\title{
Free energy of formation of a crystal nucleus in incongruent solidification: Implication for modeling the crystallization of aqueous nitric acid droplets in type 1 polar stratospheric clouds
}

\author{
Yuri S. Djikaev* and Eli Ruckenstein ${ }^{\dagger}$ \\ Department of Chemical and Biological Engineering, SUNY at Buffalo, \\ Buffalo, New York 14260
}

\begin{abstract}
.
Using the formalism of classical thermodynamics in the framework of the classical nucleation theory, we derive an expression for the reversible work $W_{*}$ of formation of a binary crystal nucleus in a liquid binary solution of non-stoichiometric composition (incongruent crystallization). Applied to the crystallization of aqueous nitric acid droplets, the new expression more adequately takes account of the effects of nitric acid vapor compared to the conventional expression of MacKenzie, Kulmala, Laaksonen, and Vesala (MKLV) [J.Geophys.Res. 102, 19729 (1997)]. The predictions of both MKLV and modified expressions for the average liquid-solid interfacial tension $\sigma^{\text {ls }}$ of nitric acid dihydrate (NAD) crystals are compared by using existing experimental data on the incongruent crystallization of aqueous nitric acid droplets of composition relevant to polar stratospheric clouds (PSCs). The predictions for $\sigma^{\text {ls }}$ based on the MKLV expression are higher by about $5 \%$ compared to predictions based on our modified expression. This results in similar differences between the predictions of both expressions for the solid-vapor interfacial tension $\sigma^{\text {sv }}$ of NAD crystal nuclei. The latter can be obtained by using the method based on the analysis of experimental data on crystal nucleation rates in aqueous nitric acid droplets; it exploits the dominance of the surface-stimulated mode of crystal nucleation in small droplets and its negligibility in large ones. Applying that method to existing experimental data, our expression for the free energy of formation provides an estimate for $\sigma^{\mathrm{sv}}$ of NAD in the range $\approx 92 \mathrm{dyn} / \mathrm{cm}$ to $\approx 100 \mathrm{dyn} / \mathrm{cm}$, while the MKLV expression predicts it in the range $\approx 95 \mathrm{dyn} / \mathrm{cm}$ to $\approx 105 \mathrm{dyn} / \mathrm{cm}$. The predictions of both expressions for $W_{*}$ become identical for the case of congruent crystallization; this was also demonstrated by applying our method for determining $\sigma^{\text {sv }}$ to the nucleation of nitric acid trihydrate (NAT) crystals in PSC droplets of stoichiometric composition.
\end{abstract}

${ }^{*}$ Corresponding author. E-mail: idjikaev@buffalo.edu

${ }^{\dagger}$ E-mail: feaeliru@buffalo.edu 


\section{Introduction}

At present, there remains no doubt that type 1 polar stratospheric clouds (PSCs) play a crucial role in the atmospheric ozone depletion in general and formation of the so-called "polar ozone holes" (dramatic springtime decrease in stratospheric ozone over polar regions) in particular. ${ }^{1,2}$ Chemical reactions on the surface of type 1 PSC particles in the wintertime polar stratosphere trigger ${ }^{3}$ a large, albeit localized and seasonal, increase in the amount of chlorine in its active, ozone-destroying radical forms. Under the springtime sunlight, such radicals efficiently destroy ozone molecules in a series of chain reactions. Type 1 PSC formation is also harmful because, through sedimentation, it removes gaseous nitric acid from the stratosphere which would otherwise combine with $\mathrm{ClO}$ to form less reactive forms of chlorine.

Since the chemical activity of type 1 PSCs strongly depends on phases present in their particles, it is important to understand both the conditions under which they form and their phase evolution. In particular, it is important to predict atmospheric conditions (temperatures, partial pressures, etc...) under which liquid droplets of type 1b PSCs freeze, thus transforming into solid particles of type 1a PSCs. A (parameterized) theoretical description of the crystallization of atmospheric droplets constitutes an integral part of the aerosol/cloud component of regional and global climate models; the improvement of the former will hence lead to the improvement of the latter and enhancement of their predictive powers.

One of the most important characteristics of the crystallization of liquids is the crystal nucleation rate. Until about 2002, crystallization in homogeneous liquids had been assumed to initiate within the volume of the supercooled phase. ${ }^{4,5}$ Under such an assumption, the rate of crystallization of a droplet is proportional to its volume. ${ }^{4-6}$ Not surprisingly, until about a decade ago, experimental data on the crystallization of aqueous nitric acid droplets had been analyzed under the assumption 
that the NAD and NAT nucleation there took place in the interior of droplets.

However, Tabazadeh et $a l .{ }^{7}$ re-examined some laboratory data on the homogeneous freezing of aqueous nitric acid droplets and suggested crystal nucleation to occur "pseudoheterogeneously" at the air-droplet interface. Similar conclusions were drawn for the freezing of pure water droplets. ${ }^{8}$

Moreover, using the classical nucleation theory (CNT), Djikaev et al. ${ }^{9,10}$ developed a thermodynamic theory of surface-stimulated nucleation. The theory prescribes the condition under which the surface of a droplet can stimulate crystal nucleation therein so that the formation of a crystal nucleus with one of its facets at the droplet surface ("surface-stimulated" mode) is thermodynamically favored over its formation with all the facets within the liquid phase ("volume-based" mode). For both unary and multicomponent droplets, this condition coincides with the condition for the partial wettability of at least one of the crystal nucleus facets by the liquid. ${ }^{11}$ This effect was experimentally observed for several systems, ${ }^{12-14}$ including water-ice ${ }^{15}$ at temperatures at or below $0^{\circ} \mathrm{C}$.

Clearly, under otherwise identical thermodynamic conditions, the free energy barrier of heterogeneous nucleation is much lower than that of homogeneous nucleation. ${ }^{6,11,16,17}$ However, as previously shown, ${ }^{18}$ surface-stimulated crystal nucleation should not be considered as a particular case of heterogeneous nucleation and its kinetics cannot be treated by using the formalism of heterogeneous nucleation on foreign surfaces (such a conclusion also transpired from some experimental results on the freezing of both pure water ${ }^{19}$ and aqueous nitric acid ${ }^{20}$ droplets); it is much more likely to be a special case of homogeneous crystal nucleation, ${ }^{18}$ where one of the facets of a crystal nucleus represents a part of the surface of the (freezing) droplet.

The free energy of formation of such a "surface-stimulated" crystal nucleus is related to the free energy of formation of a "volume-based" nucleus (with all its facets within the droplet) through an expression involving the morphology parameters of the crystal nucleus and its interfacial tensions with both liquid and vapor phases. ${ }^{18}$ Using that relationship, one can determine the solid-vapor 
interfacial tension via experiments on crystal nucleation in droplets of different sizes. The method exploits the dominance of the surface-stimulated mode of crystal nucleation in small droplets (of radii $R \lesssim 5 \mu \mathrm{m}$ ) and its negligibility in large ones (of radii $R \gtrsim 20 \mu \mathrm{m}$ ). Experimentally measuring the crystal nucleation rates in such large and small (but otherwise identical) droplets, one can determine the solid-vapor interfacial tension of that crystal facet which forms at the droplet surface in a surfacestimulated mode.

The proposed method assumes that there exists an analytical expression for the free energy of formation of a crystal nucleus within the liquid phase (i.e., in the volume-based mode). In the case where crystallization occurs in unary liquids or if a multicomponent crystalline phase forms out of its own, stoichiometric melt (solution), such an expression has been well established (in the framework of CNT) and widely used for extracting the liquid-solid interfacial tension from nucleation experiments. ${ }^{6,21-23}$

For the case where a multicomponent crystalline phase forms out of a non-stoichiometric solution (incongruent solidification), an analogous expression (relating the free energy of formation of a crystal nucleus to its solid-liquid interfacial tension) exists as well, and it is usually cited ${ }^{21,23-25}$ as due to MacKenzie, Kulmala, Laaksonen, and $\operatorname{Vesala}^{26}$ (hereinafter referred to as MKLV) and Pruppacher and Klett. ${ }^{6}$ While we were not able to locate that particular expression in the book by Pruppacher and Klett, ${ }^{6}$ MKLV provide that expression (equation (1) in ref.26) stating that its derivation “...follows Pruppacher and Klett [1978, p. 136ff, and p.152], and assumes that all solid phases are pure." Despite the latter assumption, the MKLV expression has been widely applied to crystal nucleation in aqueous solutions even when the solid phase was not pure ice; in particular, it was used to extract estimates for the crystal-liquid interfacial tension of NAD and NAT from the experiments on the freezing of droplets of aqueous nitric acid. ${ }^{21,23,25,26}$

Avoiding such an assumption (that the solid, crystallizing phase is a pure, single-component 
one), in this paper we will present the derivation of an expression for the free energy of formation of a binary crystal nucleus within a non-stoichiometric solution (incongruent crystallization), thus obtaining an expression, analogous to that of MKLV, but which more adequately takes account of the presence both components in the system, including the vapor-gas medium (wherewith the liquid phase is assumed to be in equilibrium). Applying our expression to experiments on the freezing of large aqueous nitric acid droplets, ${ }^{25}$ we will then evaluate the solid-liquid interfacial tensions of NAD crystals in non-stoichiometric solutions and compare our estimates with those, obtained on the basis

of the MKLV expression. ${ }^{26}$ Furthermore, using these estimates and applying our previously proposed method (mentioned above) to experiments on the freezing of small aqueous nitric acid droplets of non-stoichiometric composition, ${ }^{20}$ we will also evaluate the solid-vapor interfacial tensions of NAD crystals and compare the predictions based on the MKLV expression (for the free energy of formation of a crystal nucleus) with those based on our expression. As a further illustration of that method, we will also present evaluations of the vapor-solid interfacial tensions of NAT crystals obtained by analyzing the experimental data on the NAT crystallization in large $\mathrm{e}^{25}$ and small ${ }^{27}$ aqueous nitric acid droplets of stoichiometric composition.

\section{Free energy of formation of a binary crystal nucleus within a binary solution of arbitrary composition}

It should be emphasized that we are interested in only one of several possible pathways whereby liquid aqueous NA droplets may transform into solid particles of NA hydrates. Specifically, we consider the case where solid (pure) NAD or NAT particles form via homogeneous crystallization of aqueous NA droplets. Thus, (critical) crystal nuclei of NAD/NAT form homogeneously, without even ice embryos within a droplet. The possible presence of ice clusters in droplets of very dilute aqueous NA solution is likely to result in the dominance of the heterogeneous mechanism of binary nucleation 
in the freezing of NAD/NAT particles or even in the nucleation of ice and subsequent freezing of binary solutions which would lead to the phase separation and the formation of two-phase composite droplets, partly consisting of pure ice and partly of solid NAD/NAT (see Bogdan and Molina ${ }^{28}$ ).

In the framework of the classical thermodynamics, an expression for the reversible work of homogeneous formation of a binary crystal nucleus within a liquid binary solution can be derived following the procedure, used in ref.10 to study the effects of adsorption and dissociation on the thermodynamics of surface-stimulated crystal nucleation in multicomponent droplets. In that procedure, the liquid solution (whether bulk or in droplets) is assumed to remain in equilibrium with the corresponding vapor mixture at all times.

If the chemical potentials of all the components in the vapor phase are fixed, so are those in the liquid solution. Under such conditions, the composition of the liquid solution remains constant during crystal nucleation. Often, however, a liquid solution freeze into a solid of fixed composition, that is independent of a variable solution composition. (For example, nitric acid hydrates can crystallize from an aqueous nitric acid solution of variable composition.) If the composition of the liquid solution differs from that of the solid phase, material must be exchanged between the vapor mixture and the liquid solution to maintain equilibrium. This exchange of material can lead to a change in the vapor and liquid volumes (note that the total volume of the system is fixed). Thus, unlike the case of crystallization in single-component liquids, ${ }^{9}$ it is now necessary to consider the vapor mixture as a part of our system ${ }^{10}$ in order to take into account the possibility of formation of a solid phase of fixed composition from a multicomponent solution of arbitrary (non-stoichiometric) composition.

It should be noted, however, that we are interested only in the nucleation stage of the crystallization of droplets (without considering the stage of crystal growth), which ends much earlier than any phase separation effects (such as those studied, e.g., by Bogdan et al. ${ }^{28}$ and Bogdan and Molina ${ }^{29}$ ) take place. Indeed, at this (nucleation) stage, the sub-critical and near-critical crystal clusters are 
so small compared to droplet sizes, that the formation of a crystal nucleus has virtually no effect on the composition of even relatively very small droplets of type 1b PSCs. For example, consider a droplet of radius $10 \mathrm{~nm}$ with the mole fraction of NA $\chi=0.3$; such a droplet will contain about 38511 molecules total (11553 molecules of NA and 26958 molecules of water). If there forms a crystal nucleus of NAD (with $\chi^{\delta}=(1 / 3) \neq \chi$ ) consisting of 30 molecules of water and 60 molecules of NA (typical sizes of crystal nuclei, according to refs.6, 11, 16) in such a droplet, the composition of its liquid part will become $\chi \approx 0.299922$, changing by less than $0.03 \%$, i.e., remaining virtually constant. Therefore, it is reasonable to assume that at the crystal nucleation stage the composition of the liquid droplet remains virtually constant even if its composition is markedly non-stoichiometric; hence, no phase separation can occur upon the formation of a single crystal nucleus.

Thus, for convenience, the work of formation can be calculated in the constant $N, V, T$ ensemble, where $N$ is the total number of molecules in the system, $V$ is its volume, and $T$ is the temperature (assumed to be uniform in the system, including the droplet). Strictly speaking, in this ensemble it is not possible to fix the chemical potentials of the vapor mixture, since its volume and number of molecules can vary during crystallization. However, as clear from the foregoing, the variations of the chemical potentials that might arise at the nucleation stage during incongruent crystallization, will be rather negligible. Likewise, the change in the volume of the liquid droplet due to the formation of a tiny crystal nucleus therein can be also neglected owing to small differences in the densities of liquid and solid phases (and smallness of the nucleus compared to the droplet). Moreover, in the thermodynamic limit, since the crystalline nucleus is usually very small compared to the bulk liquid solution, the relative inaccuracy in the free energy due to such variations tends to zero, and the reversible work of formation of a crystal particle does not depend on the choice of the ensemble (for more detailed discussion of this issue see, e.g., refs.9 and 10). The equivalence of different thermodynamic potentials (Helmholtz free energy, Gibbs free energy, and grand thermodynamic 
potential) in calculating the free energy of formation of a nucleus of a new phase was first proven by Lee et al. $^{30}$ (see section 2 therein).

Consider a liquid binary solution (either in a container or in a droplet) with a binary crystal particle formed therewithin. The crystal is of arbitrary shape with $\lambda$ facets. We will use the Greek superscripts $\alpha, \beta$, and $\delta$ to mark quantities for the liquid, vapor, and crystal, respectively, while double Greek superscripts mark quantities at the corresponding interfaces.

Assuming the crystallization process to be isothermal (with the constant uniform temperature $T$ below the temperature $T_{m}$ of solid-liquid phase equilibrium at given partial pressures $)^{9,10}$ and taking into account the foregoing arguments, the reversible work of crystal nucleus formation, $W$, can be determined via the difference between $F_{\text {fin }}$, the Helmholtz free energy of the system in its final state (vapor+liquid+crystal), and $F_{i n}$, the Helmholtz free energy in the initial state (vapor+liquid):

$$
W=F_{\text {fin }}-F_{\text {in }} .
$$

The negligibility of non-isothermal effects during crystal nucleation in droplets is a reasonable assumption for both laboratory and atmospheric conditions. ${ }^{6,11,16}$

Using the formalism of CNT (based on the capillarity approximation in the framework of the classical thermodynamics) and taking into account both dissociation of solute molecules in the solution and surface adsorption effects, one can obtain ${ }^{10}$ the following expression for the reversible work of homogeneous formation of a binary crystal particle of a fixed composition within a non-stoichiometric liquid binary solution of different (arbitrary) composition (incongruent solidification):

$$
\begin{aligned}
W= & -\sum_{i} \nu_{i}\left(\Delta H_{i} \ln \Theta-k_{B} T z_{i}^{\alpha} \ln \frac{a_{i}^{\delta}\left(\chi^{\delta}\right)}{a_{i}^{\alpha}\left(\chi^{\alpha}\right)}\right)+\sum_{i} \sum_{j=1}^{\lambda} N_{i j}^{\alpha \delta}\left(z_{i j}^{\alpha \delta} \mu_{i j}^{\alpha \delta}\left(\Gamma_{j}^{\alpha \delta}, T\right)-z_{i}^{\alpha} \mu_{i}^{\alpha}\left(P^{\alpha}, \chi^{\alpha}, T\right)\right) \\
& +\sum_{j=1}^{\lambda} \sigma_{j}^{\alpha \delta} A_{j}^{\alpha \delta} .
\end{aligned}
$$

Here, $\nu_{i}, \chi_{i}=\nu_{i} /\left(\nu_{1}+\nu_{2}\right)$, and $\mu_{i}(P, \chi, T)$ are the number of molecules, mole fraction, and chemical 
potential of component $i \quad(i=1,2)$, respectively (clearly, the composition of a binary system is uniquely defined by $\left.\chi \equiv \chi_{1}\right) ; P$ is the pressure, and $\Theta \equiv T / T_{m}$. The first term on the RHS of eq.(2) represents the excess Gibbs free energy of the molecules in the bulk crystal with respect to their free energy in the liquid state, and is related to the partial molecular enthalpy of fusion of all components $\Delta H_{i} \equiv \Delta H_{i}\left(\chi^{\delta}\right)<0$ for the case in which the solid and liquid phases have the same composition (congruent melting/solidification). The numbers of ions into which a molecule of component $i$ dissociates in the solution and (when adsorbed) at the facet $j$ are denoted by $z_{i}^{\alpha}$ and $z_{i j}^{\alpha \delta}$, respectively. For components that dissociate in the solution or at the interface, the corresponding chemical potentials, $\mu$ 's, and activities, $a$ 's, should be understood as mean ionic chemical potentials and mean activities, respectively. The second term on the RHS of eq.(2) is due to the adsorption of both components at the facets of the crystal particle. The surface area and surface tension of the facet $j$ are denoted by $A_{j}$ and $\sigma_{j}$, respectively. The total number of molecules, chemical potential, and adsorption of component $i$ at facet $j$ are denoted by $N_{i j}, \mu_{i j}$, and $\Gamma_{i j}$, respectively. The composite variable $\Gamma_{j}^{\alpha \delta}$ is the pair of adsorptions $\left\{\Gamma_{1 j}^{\alpha \delta}, \Gamma_{2 j}^{\alpha \delta}\right\}$ of both components, with $\Gamma_{i j}^{\alpha \delta}=N_{i j}^{\alpha \delta} / A_{j}^{\alpha \beta}(i=$ $1,2)$.

Note that eq.(2) takes into account the density difference between crystal and liquid phases. On the other hand, it assumes that in the temperature range between $T$ and $T_{m}$ the partial enthalpy of fusion $\Delta H_{i}$ does not change significantly. If this assumption is not accurate enough, then $\Delta H_{i}$ must be interpreted as some average value between temperatures $T$ and $T_{0}$ (such as, e.g., $\Delta H_{i}=$ $\left(\Delta H_{i}\left(T_{m}\right)+\Delta H_{i}(T)\right) / 2$, in the simplest approximation).

Equation (2) allows the composition of the solid phase to differ from that of the liquid solution. Thus during crystallization the vapor mixture and liquid solution have to continually adjust the equilibrium between them if $\chi^{\alpha}$ differs from $\chi^{\delta}$ (as is often the case with freezing of aqueous nitric acid into nitric acid hydrates). Since we work in the closed $N V T$ ensemble, the composition of the 
liquid solution may change as it freezes, and so may the vapor (and hence the liquid) pressures. In addition, the density of the liquid may differ from that of the solid. If it does, the crystal formation will induce a change in the volume of the vapor phase. The volume change can affect the partial pressures in the vapor mixture (and the pressure in the liquid), which in turn can induce a change in the liquid composition. However, all the above changes can be neglected at the stage of crystal nucleation where the sizes of crystal particles are still so small

Introduce a characteristic linear size ("radius") $r$ and a characteristic surface tension $\sigma^{\alpha \delta}$ for a crystal surrounded by its melt as

$$
r=\left[\frac{3}{4 \pi} \sum \nu_{i} v_{i}^{\delta}\right]^{1 / 3}, \quad \sigma^{\alpha \delta}=\frac{1}{4 \pi R^{2}} \sum_{j=1}^{\lambda} \sigma_{j}^{\alpha \delta} A_{j}^{\alpha \delta},
$$

where $v_{i}^{\delta}$ is the partial volume per molecule of component $i$ in the solid phase. If the crystal had a spherical shape, it would have a radius $r$ and a surface tension $\sigma^{\alpha \delta}$.

As usual, define a critical crystal (nucleus) as a crystal that is in unstable equilibrium with the surrounding fluid. The linear size and composition of such a crystal nucleus can be found by solving simultaneous equations (representing the conditions for the extremum of the free energy of formation of a critical crystal, i.e., nucleus $)^{4,6,10,11,16-18}$

$$
\partial W /\left.\partial \nu_{i}\right|_{r_{*}, \chi_{*}}=0 \quad(i=1,2)
$$

(with $\chi_{*}$ bound to be equal to $\chi^{\delta}$ when the solid phase can have only specific stoichiometric composition, as in the case of formation of NAD or NAT crystals). (hereafter the subscript "** indicates quantities corresponding to the nucleus).

Substituting eq.(2) into eq.(4), recalling that $\nu_{i}, N_{i j}^{\alpha \delta} \quad(i=1,2 ; j=1, \ldots, \lambda)$ are all independent variables of state of the crystal particle, and taking account of definitions (3), one can show that the characteristic radius $r_{*}$ of the crystal nucleus is ${ }^{10}$

$$
r_{*}=2 \sigma^{\alpha \delta} v_{i}^{\delta}\left(\Delta H_{i}\left(\chi^{\delta}\right) \ln \Theta-k_{B} T z_{i} \ln \frac{a_{i}^{\delta}\left(\chi_{i}^{\delta}\right)}{a_{i}^{\alpha}\left(\chi_{i}^{\alpha}\right)}\right)^{-1} \quad(i=1,2) .
$$


Alternatively, one can rewrite this expression in a more symmetric form,

$$
r_{*}=2 \sigma^{\alpha \delta} \bar{v}^{\delta}\left(\Delta q\left(\chi^{\delta}\right) \ln \Theta-k_{B} T\left(\chi^{\delta} z_{1} \ln \frac{a_{1}^{\delta}\left(\chi^{\delta}\right)}{a_{1}^{\alpha}\left(\chi^{\alpha}\right)}+\left(1-\chi^{\delta}\right) z_{2} \ln \frac{a_{2}^{\delta}\left(\chi^{\delta}\right)}{a_{2}^{\alpha}\left(\chi^{\alpha}\right)}\right)\right)^{-1}
$$

where $\Delta q=\chi^{\delta} \Delta H_{1}+\left(1-\chi^{\delta}\right) \Delta H_{2}$ and $\bar{v} \equiv \bar{v}^{\delta}=\chi^{\delta} v_{1}^{\delta}+\left(1-\chi^{\delta}\right) v_{2}^{\delta}$.

From this point on, let us assign subscript "1" to nitric acid (NA) and replace it by "na", while assigning subscript "2" to water and replacing it by "w". Since the work of crystal formation $W$ as a function of $r$ attains its maximum at $r=r_{*}$, one can then obtain

$$
W_{*}=\frac{(16 \pi / 3)\left(\sigma^{l s}\right)^{3} \bar{v}^{2}}{\left[\Delta q \ln \left(T / T_{m}\right)+k_{B} T\left(\chi^{\delta} \ln \frac{P_{n a}^{e}(\chi)}{P_{n a}^{e}\left(\chi^{\delta}\right)}+\left(1-\chi^{\delta}\right) \ln \frac{P_{w}^{e}(\chi)}{P_{w}^{e}\left(\chi^{\delta}\right)}\right)\right]^{2}},
$$

where $\chi$ and $\chi^{\delta}$ are the mole fractions of solute molecules in the liquid solution and solid (crystalline) phase, respectively; $P_{n a}^{e q}\left(\chi^{\prime}\right)$ and $P_{w}^{e q}\left(\chi^{\prime}\right)$ are the equilibrium vapor pressures of NA and water, respectively, at a given temperature $T$ over a bulk liquid binary solution of composition $\chi^{\prime}$ (mole fraction of component 1).

Deriving eq.(7), it was taken into account that at melting temperature $T_{m}$, the activity coefficient of each component is the same in both phases for congruent melting, i.e., $a_{i}^{\alpha}\left(\chi^{\delta}, T_{m}\right)=$ $a_{i}^{\delta}\left(\chi^{\delta}, T_{m}\right) \quad(i=1,2)$. Besides, they were assumed to be weakly dependent on temperature in the vicinity of $T_{m}$, i.e., $\partial a_{i}^{\alpha}\left(\chi^{\delta}, T\right) / \partial T \ll a_{i}^{\alpha}\left(\chi^{\delta}, T_{m}\right) /\left|T-T_{m}\right|$.

Taking into account the foregoing and comparing eq.(7) with the MLKV expression for $W_{*}$,

$$
W_{*}=\frac{(16 \pi / 3)\left(\sigma^{l s}\right)^{3} \bar{v}^{2}}{\left[\Delta q \ln \left(T / T_{m}\right)+k_{B} T \ln \frac{P_{w}^{e}(\chi)}{P_{w}^{e}\left(\chi^{\delta}\right)}\right]^{2}},
$$

(eq.(1) in ref.26), it is clear that the principal difference between the two is the absence of the term $k_{B} T\left(\chi^{\delta}\right) \ln \frac{P_{n a}^{e}(\chi)}{P_{n a}^{e}\left(\chi^{\delta}\right)}$ from the denominator on the RHS of eq.(8), representing the ratio of the vapor pressures of nitric acid in equilibrium with the actual solution and with the solution of stoichiometric composition (equal to that of the solid phase). This is not surprising, because, as explicitly stated by MKLV ${ }^{26}$ their expression, equation (8), assumes that the solid phase was a pure solid (ice). What is 
surprising is that eq.(8) was applied to the formation of NAD/NAT crystals in aqueous NA droplets of non-stoichiometric composition. ${ }^{21,23,25,26}$ It should be noted, that the original expression for $W_{*}$ by MacKenzie et al. (ref. 22, eq.(13)) was more adequate (except for some typos therein) for incongruent solidification than their corrected version, ${ }^{26}$ eq.(8) above. On the other hand, eq.(8) (i.e., equation (1) in ref.26) would be correct if, in the denominator of its RHS, the enthalpy of freezing had been replaced by the partial molar enthalpy of freezing of water and, in the numerator of its RHS, the average volume per molecule $\bar{v}$ had been replaced by the partial molecular volume of water molecules $v_{w}^{\delta} \equiv v_{w}\left(\chi^{\delta}\right)$ in the solid phase of composition $\chi^{\delta}$. (As a side note, $\bar{v}$ in the numerator of eq.(8) should be the average molecular volume in the solid phase rather than in the liquid; however, the differences in $\bar{v}$ for the solid and liquid phases can be neglected in most atmospheric applications.)

Since both equations (7) and (8) assume the crystal nucleus to be spherical, they allow one determine $\sigma^{l s}$, average (over all crystal facets) liquid-solid interfacial tension of the nucleus in a solution of arbitrary composition if $W_{*}$ is known. Equation (7) provides

$$
\sigma^{l s}=2 W_{*} \bar{v}\left[\Delta q \ln \left(T / T_{m}\right)+k_{B} T\left(\chi^{\delta} \ln \frac{P_{n a}^{e}(\chi)}{P_{n a}^{e}\left(\chi^{\delta}\right)}+\left(1-\chi^{\delta}\right) \ln \frac{P_{w}^{e}(\chi)}{P_{w}^{e}\left(\chi^{\delta}\right)}\right)\right]^{-1} .
$$

whereas eq.(8) leads to

$$
\sigma^{l s}=2 W_{*} \bar{v}\left[\Delta q \ln \left(T / T_{m}\right)+k_{B} T \ln \frac{P_{w}^{e}(\chi)}{P_{w}^{e}\left(\chi^{\delta}\right)}\right]^{-1}
$$

The free energy of formation of a crystal nucleus can be obtained by matching experimental crystal nucleation rates $J^{\exp }$ with theoretical predictions $J_{v}$ of CNT, i.e., by solving the equation $J^{\exp }=J_{v}$ with respect to $W_{*}$, with

$$
J_{v}=\frac{k_{B} T}{h} \rho_{l} \mathrm{e}^{-\Delta G_{d} / k_{B} T} \mathrm{e}^{-W_{*} / k_{B} T},
$$

where $k_{B}$ and $h$ are the Boltzmann and Planck constants, $T$ is the absolute temperature, $\rho_{l}$ is the number density of liquid molecules, where $\Delta G_{d}$ is the activation energy for the diffusion of one 
molecular unit from the bulk liquid to the solid (often called the diffusion-activation energy), and $W_{*}$ is the reversible work of formation of a crystal nucleus within the liquid. Thus,

$$
W_{*}=-\Delta G_{d}-k_{B} T \ln \left[\frac{h}{k_{B} T} \frac{J^{\mathrm{exp}}}{\rho_{\mathrm{liq}}}\right] \text {. }
$$

\section{Two modes of crystal nucleation in droplets}

Equation $J^{\exp }=J_{v}$, used above, implies that homogeneous crystal nucleation in droplets is exclusively volume-based, i.e., that all crystal nuclei are within the liquid. However, as mentioned above, it was shown both theoretically $9,10,18$ and via analysis ${ }^{7,8}$ of existing experimental data that under some conditions the surface of a droplet can stimulate crystal nucleation therein so that the formation of a crystal nucleus with one of its facets at the droplet surface ("surface-stimulated" mode) is thermodynamically favored over its formation with all the facets within the liquid phase ("volumebased" mode). For both unary and multicomponent droplets, this condition coincides with the condition for the partial wettability of at least one of the crystal nucleus facets by the liquid. ${ }^{6,11}$

Neglecting the contributions from line tensions, the criterion for whether crystal nucleation in a supercooled droplet will or will not be thermodynamically stimulated by the surface it has the form $^{9,10}$

$$
\sigma_{\lambda}^{s v}-\sigma^{l v}<\sigma_{\lambda}^{l s}
$$

where $\sigma^{l v}$ the liquid-vapor surface tension, and $\sigma_{\lambda}^{l s}$ and $\sigma_{\lambda}^{s v}$ are the interfacial tensions of crystal facet $\lambda$ in the liquid and in the vapor, respectively.

Taking this into account, it was suggested ${ }^{7,8,18}$ that the total crystal nucleation rate $J$ in experiments on droplet freezing can contain two contributions, one due to the volume-based crystal nucleation and the other to the surface-stimulated mode. The surface-stimulated mode of crystallization can become important when the crystallizing liquid is in a dispersed state, which is the case with the freezing of atmospheric droplets and many experiments. Indeed, since smaller droplets have a 
higher surface-to-volume ratio than larger ones, nucleation rates in the former will be higher than in the latter (or in bulk). Hence it is experimentally easier to observe the crystallization in an ensemble of small aerosols than of large ones, assuming the total liquid volume is the same in both cases.

Furthermore, it was argued ${ }^{18}$ that the formation of a crystal nucleus with one of its facets at the droplet surface cannot start preferentially at the surface, because the latter does not have any sites which would make the ordering of the surrounding surface molecules thermodynamically more favorable than the ordering of interior molecules. On the contrary, the surface layer of a crystal remains disordered far below the melting temperature due to weaker constraints on the surfacelocated molecules which have a reduced number of neighbors and hence have a higher vibrational amplitude compared to bulk ones. This results in the formation of a thin disordered layer on the crystal surface at temperatures significantly lower than the melting one. This phenomenon, referred to as premelting, has been well established both experimentally and via molecular dynamics simulations (see ref.31 and references therein). Moreover, it was experimentally observed ${ }^{32,33}$ that the premelting of the (0001) face of hexagonal ice occurs at about $200 \mathrm{~K}$, far below the lowest temperature reported for homogeneous freezing of atmospheric droplets.

Thus, one can conclude that a crystal nucleus with one facet as a droplet-vapor interface cannot begin its formation (as a subcritical crystal) at the droplet surface. ${ }^{18}$ Still, the surface of the droplet can stimulate crystal nucleation therein (under condition (12)), but a crystal cluster has to begin its initial evolution homogeneously in a spherical layer adjacent to the droplet surface ("sub-surface layer"). When this crystal becomes large enough (due to the fluctuational growth usual for nucleation), one of its facets hits the droplet surface and at this moment or shortly thereafter it becomes a nucleus owing to a drastic change in its thermodynamic state. ${ }^{18}$ any crystalline cluster, starting its evolution with its center in the "surface-stimulated nucleation" layer, has a potential to become a nucleus (by means of fluctuations) once one of its facets, satisfying eq.(1), meets the droplet sur- 
face. Originally proposed in ref.18, this idea was shown to be plausible in later experiments ${ }^{19}$ and molecular dynamics simulations. ${ }^{34}$

In this model, the total per-droplet rate of crystal nucleation is the sum of the contributions from both volume-based and surface-stimulated modes, $J V_{R}=J_{v}^{s} V_{R}^{s}+J_{v}\left(V_{R}-V_{R}^{s}\right)$, where $J_{v}^{s}$ is the number of crystal nuclei, forming in a surface-stimulated mode per unit time in unit volume of the surface-stimulated nucleation layer (of total volume $V_{R}^{s}$ ), $J_{v}$ is the rate of volume-based nucleation, and $V_{R}$ is the volume of the droplet. In the framework of CNT,

$$
J_{v}^{s}=\frac{k_{B} T}{h} \rho_{l} \mathrm{e}^{-\Delta G_{d} / k_{B} T} \mathrm{e}^{-\widetilde{W}_{*} / k_{B} T},
$$

where the density of molecules in the droplet is assumed to be uniform up to the dividing surface, and $\widetilde{W}_{*}$ is the work of formation of a surface-stimulated crystal nucleus. Introducing a variable $u=\left(\sigma_{\lambda}^{s v}-\sigma^{l v}\right) / \sigma_{\lambda}^{l s}$, for the total rate of crystal nucleation one can obtain ${ }^{18}$

$$
J=\left[1+\left(1-\left(1-\left(h_{\lambda} / R\right) u\right)^{3}\right)\left(\mathrm{e}^{-\frac{1}{2}(u-1) W_{*} / k_{B} T}-1\right)\right] J_{v}
$$

where $h_{\lambda}$ is the height of a pyramid whereof the apex is at the center of the volume-based crystal nucleus and the basis is the facet $\lambda$, satisfying eq.(12).

Using eq.(18) from ref.10, one can show, that independent of the shape of the crystal nucleus, its volume can be found as

$$
V_{*}=2 W_{*} \bar{v}\left[\Delta q \ln \left(T / T_{m}\right)+k_{B} T\left(\chi^{\delta} \ln \frac{P_{n a}^{e}(\chi)}{P_{n a}^{e}\left(\chi^{\delta}\right)}+\left(1-\chi^{\delta}\right) \ln \frac{P_{w}^{e}(\chi)}{P_{w}^{e}\left(\chi^{\delta}\right)}\right)\right]^{-1} .
$$

Adopting a more realistic assumption (compared to the assumption of sphericity) that the crystal nucleus has a shape of a right prism with an arbitrary aspect ratio $\kappa$, and assuming that the basal facets of the prism are those that satisfy the criterion of partial wettability, eq.(10), the quantity $h_{\lambda}$ (defined above) will be equal to the half-height of the prism. Thus, one can obtain

$$
h_{\lambda}=\left(\kappa^{2} V_{*}\right)^{1 / 3} / \sqrt{3} .
$$


For large droplets of radii $R \gtrsim 20 \mu \mathrm{m}$, the RHS of eq.(13) reduces ${ }^{18}$ to $J_{v}$. Thus, if crystal nucleation rates $J_{\text {Large }}^{\text {exp }}$ are measured in experiments on large droplets, one can find $W_{*}$ (from $J_{\text {Large }}^{\exp }=$ $J_{v}$ ), and then extract an average value $\sigma^{l s}$ from eq.(9). If, under identical experimental conditions, crystal nucleation rates $J_{\text {small }}^{\text {exp }}$ are measured in experiments on small droplets (of radius $R \lesssim 5 \mu \mathrm{m}$ ), then the rates $J_{\text {small }}^{\exp }$ and $J_{\text {Large }}^{\exp }$ will be related as

$$
\frac{J_{\text {small }}^{\text {exp }}}{J_{\text {Large }}^{\text {exp }}}=\left[1+\left(1-\left(1-\left(h_{\lambda} / R\right) u\right)^{3}\right)\left(\mathrm{e}^{-\frac{1}{2}(u-1) W_{*} / k_{B} T}-1\right)\right]
$$

Since $W_{*}$ can be extracted from experiments on freezing of large droplets, so can $\sigma^{l s}$ and $h_{\lambda}$. Therefore, equation (16) can be solved with respect to $u$. Assuming that $\sigma^{l v}$ is known and that $\sigma_{\lambda}^{l s} \approx \sigma^{l s}$, one can determine the solid-vapor interfacial tension $\sigma_{\lambda}^{s v}$ of the facet $\lambda$ as

$$
\sigma_{\lambda}^{s v}=\sigma^{l v}+u_{0} \sigma_{\lambda}^{l s}
$$

where $u_{0}$ is the (physically meaningful) solution of equation (16).

Clearly, this method for determining the solid-vapor interfacial tension $\sigma_{\lambda}^{s v}$ from experiments on the freezing of aqueous NA droplets, requires the experimental data in for crystal nucleation rates in large and small droplets, $J_{\text {Large }}^{\text {exp }}$ and $J_{\text {small }}^{\text {exp }}$, to be for identical experimental conditions, droplets differing only in their size. Otherwise, its application provide only rough estimates for $\sigma_{\lambda}^{s v}$.

The RHS of eq.(16) is a non-monotonic function of $u, f(u)$ (Figure 1), hence eq.(16) can formally have two roots: one, $u^{\prime}$, with $d f(u) /\left.d u\right|_{u^{\prime}}>0$, and the other, $u_{0}$, with $d f(u) /\left.d u\right|_{u_{0}}<0$. However, the root $u^{\prime}$ is not physically meaningful. Indeed, at constant $\sigma_{l v}$ and $\sigma_{\lambda}^{l s}$, one can expect $J_{\text {small }}^{\exp }$ to decrease with increasing $\sigma_{\lambda}^{s v}$ hence, $d f(u) / d u$ must be negative.

Note that, while $\sigma^{l v}, \sigma^{l s}$, and $\sigma_{\lambda}^{l s}$ depend on the composition of the solution in droplets, $\sigma_{\lambda}^{s v}$ can be expected to be independent of whether NAD/NAT crystals form in small droplets of aqueous nitric acid of stoichiometric or non-stoichiometric composition, assuming that, in both cases, the same crystal facet $\lambda$ forms as a part of the droplet-air interface. 


\section{Numerical Evaluations}

In applications to the crystallization of aqueous nitric acid droplets, the new expression more adequately takes account of the effects of nitric acid vapors compared to the conventional, widely used expression of MacKenzie, Kulmala, Laaksonen, and Vesala (MKLV). ${ }^{26}$ The predictions of both our modified expression and the MKLV one for the average liquid-solid interfacial tension $\sigma^{\text {ls }}$ of nitric acid dihydrate (NAD) crystals are then compared by applying them to the analysis of existing experimental data on the incongruent crystallization of aqueous nitric acid droplets of composition relevant to polar stratospheric clouds. It is shown that predictions for $\sigma^{\text {ss }}$ based on the MKLV expression are usually higher by about $5 \%$ compared to predictions based on our modified expression. These differences are then transferred into the predictions of both expressions for the solid-vapor interfacial tensions of NAD crystal nuclei. The latter can be obtained by using the method, that we previously developed for determining the solid-vapor interfacial tension $\sigma^{\text {sv }}$ of nitric acid dihydrate/trihydrate (NAD/NAT) crystals using experiments on crystal nucleation in aqueous nitric acid (NA) droplets; it exploits the dominance of the surface-stimulated mode of crystal nucleation in small droplets (of radii $R \lesssim 5 \mu \mathrm{m}$ ) and its negligibility in large ones (of radii $R \gtrsim 20 \mu \mathrm{m}$ ). Applying our method to existing experimental data, our expression for the free energy of formation provides an estimate for $\sigma^{\mathrm{sv}}$ of NAD to be about $90 \mathrm{dyn} / \mathrm{cm}$, while the MKLV expression predicts it to be about $95 \mathrm{dyn} / \mathrm{cm}$. The predictions of both expressions for $W_{*}$ become identical for the case of congruent crystallization; this was also demonstrated by applying our method to the nucleation of nitric acid trihydrate (NAT) crystals in droplets of stoichiometric composition.

To numerically illustrate differences between the two expressions for $W_{*}$, MKLV one (eq.(8)) and modified one (eq.(7)), one can compare the corresponding estimates for the liquid-solid and vaporsolid interfacial tensions that can be extracted from the experiments on the freezing of aqueous 
nitric acid droplets by using these expressions. An MKLV estimate $\sigma_{\mathrm{MKLV}}^{l s}$ for $\sigma_{l s}$ is provided by eq.(10), whereas a modified estimate $\sigma_{\mathrm{DR}}^{l s}$ is given by eq.(9); using them in eq.(17), one can obtain the corresponding estimates $\sigma_{\mathrm{MKLV}}^{s v}$ and $\sigma_{\mathrm{DR}}^{s v}$ for $\sigma^{s v}$.

Estimates for $\sigma_{l s}$ were obtained from the experimental data of Salcedo et al. ${ }^{25}$ for the NAD and NAT crystal nucleation rates in large aqueous nitric acid droplets (of characteristic radii about $25 \mu \mathrm{m}$ and various compositions) at stratospherically relevant temperatures. For temperatures and droplet compositions, at which experiments were not carried out, we used the parameterized expression for the crystal nucleation rates of NAD and NAT as functions of $T$ and $\mathrm{wt} \% \mathrm{HNO}_{3}$ in droplets.

For the dependence of $P_{n a}^{e q}$ and $P_{w}^{e q}$ on the temperature and composition of aqueous nitric acid solution we used analytical expressions proposed by Luo et al. ${ }^{35}$ to fit their own experimental results. The dependence of density $\rho_{l i q}$ of nitrate ions in the solution on the temperature and composition of the solution was taken from ref.36; for the stoichiometric solution at $193 \mathrm{~K} \rho_{l i q}$ was estimated to be about $9.2 \times 10^{21} \mathrm{~cm}^{-3}$, and $\bar{v}$ to be about $65.5 \mathrm{~cm}^{3} / \mathrm{mol}$, both based ${ }^{13}$ on a solution density of $1.52 \mathrm{~g} / \mathrm{cm}^{3}$. The latent heat of crystallization was taken to $\mathrm{be}^{21,37} \Delta H \approx-4.8 \mathrm{kcal} / \mathrm{mol}$. The temperature and composition dependence of the liquid-vapor surface tension $\sigma^{l v}$ and the temperature dependence of $\Delta G_{d}$ were taken from refs.38 and 24 , respectively.

Figures 2 and 3 present the $T$ - and $\chi$-dependence, respectively, of the effecitve liquid-solid interfacial tension $\sigma_{\lambda}^{l s}$ of NAD crystal nuclei obtained by using the parameterized dependence of $J_{\text {Large }}^{\text {exp }}$ on $\mathrm{T}$ and wt $\% \mathrm{HNO}_{3}$, reported in ref.25 for large aqueous NA droplets. In Fig.2, $\sigma_{\lambda}^{l s}$ is shown as a function of temperature $T$ at the mole fraction of $\mathrm{HNO}_{3}$ in the droplet a) $\chi=0.20$ and b) $\chi=0.30$, whereas in Fig. $3 \sigma_{\lambda}^{l s}$ is plotted as a function of $\chi$, at a) $T=190 \mathrm{~K}$ and b) $T=197 \mathrm{~K}$. In both Figures 2 and 3 , the solid and dashed curves correspond to $\sigma_{\mathrm{DR}}^{l s}$ and $\sigma_{\mathrm{MKLV}}^{l s}$, respectively.

As seen in Fig.2, at lower $\chi$ 's, in the temperature range from $188 \mathrm{~K}$ to $198 \mathrm{~K}$ both $\sigma_{s v}^{\mathrm{DR}}$ and $\sigma_{s v}^{\mathrm{MKLV}}$ monotonically decrease with increasing temperature, whereas for higher $\chi^{\prime}$ s they both first increase 
with increasing $T$, attain a maximum at about 192-193 K, then decrease. On the other hand (as seen in both Figs.2 and 3), the difference between them increases with increasing temperature. However, as expected, this difference becomes zero when the composition of the liquid solution reaches the stoichiometric composition of the solid phase NAD of $\chi^{\delta} \approx 0.333 \ldots$ (compositions $\chi>\chi^{\delta}$ are not shown in Fig.3 because they are irrelevant to aqueous NA droplets in PSCs under normal stratospheric conditions). One can also notice (Fig.3), that for $\chi<\chi^{\delta}, \sigma_{\mathrm{DR}}^{l s}$ is an increasing function of $\chi$, while $\sigma_{\text {MKLV }}^{l s}$ is a non-monotonic function of $\chi$ (first increasing, then decreasing with increasing $\chi$ ).

So far, the morphology of NAD and NAT crystal nuclei has not investigated thoroughly, although some interesting studies have been reported. The in-situ Fourier transform infrared (FTIR) extinction spectra of airborne $\alpha$-NAD (low-temperature modification of NAD) microparticles, formed via homogeneous nucleation in supercooled $\mathrm{HNO}_{3} / \mathrm{H}_{2} \mathrm{O}$ solution droplets, ${ }^{39}$ suggested that the observed $N A D$ crystals are highly aspherical; modeling them as prolate or oblate spheroids, the predominance of oblate shapes with the aspect ratio (defined as the ratio of the non-rotational and rotational axes of the ellipse) in the range from 6 to 20 was reported. ${ }^{39}$ The morphology of NAT crystals forming in aqueous nitric acid solutions of different compositions was studied ${ }^{40}$ by means of time-dependent X-ray powder diffraction; the shape and habit of NAT crystals were found to vary from platelet-like ones to needle-like ones, depending on the temperature and composition of the solution. To take this uncertainty into account, we assumed that NAD crystal nuclei have a shape of a right prism $(\lambda$ assumed to be the basal facet of the prism) and evaluated $\sigma_{\lambda}^{s v}$ for various values of aspect ratio $\kappa$ $(=1,10,20,30,40,50)$ in eq.(15). Our numerical evaluations of $\sigma_{\lambda}^{s v}$ are presented in Figures 4 and 5.

Figure 4 shows the $\kappa$-dependence of the solid-vapor interfacial tension $\sigma_{\lambda}^{s v}$ of facet $\lambda$ of NAD crystal nuclei obtained by using the experimental data from ref.25 (large aqueous NA droplets of nonstoichiometric composition) and ref.20 (small aqueous NA droplets of non-stoichiometric composition) for different temperatures. The results in Fig.4a were obtained by using eq.(9) for $\sigma_{l s}$ (corresponding 
to the modified expression for $W_{*}$, eq.(7)), whereas those in Fig.4b were obtained by using eq.(10) for $\sigma_{l s}$ (corresponding to the MKLV expression for $W_{*}$, eq.(8)). In both Figures $4 \mathrm{a}$ and $4 \mathrm{~b}$, circles represent data due to the experiments on the freezing of aqueous NA droplets of mean diameter 225 $\mathrm{nm}$ and NA mole fraction 0.27 at temperature $195.8 \mathrm{~K}$; squares are for droplets of mean diameter 255 $\mathrm{nm}$ and NA mole fraction 0.27 at temperature $192.2 \mathrm{~K}$; diamonds are for droplets of mean diameter $255 \mathrm{~nm}$ and NA mole fraction 0.28 at temperature $192.1 \mathrm{~K}$.

Figure 5 presents the $\kappa$-dependence of the solid-vapor interfacial tension $\sigma_{\lambda}^{s v}$ of facet $\lambda$ of NAT crystals obtained by using the experimental data of ref.25 (large aqueous NA droplets of mean radius $25 \mu \mathrm{m}$ and stoichiometric NAT compositions, $54 \mathrm{wt} \% \mathrm{HNO}_{3}$ ) and ref.27 (small aqueous NA droplets of mean radius $0.38 \mu \mathrm{m}$ and stoichiometric NAT composition of $\left.53.7 \mathrm{wt} \% \mathrm{HNO}_{3}\right)$. The circles correspond to the experiments ${ }^{27}$ at temperature $163.5 \mathrm{~K}$, the squares at $165.5 \mathrm{~K}$, and diamonds at $167 \mathrm{~K}$. One can notice that at $T=167 \mathrm{~K}$, the data points for $\kappa<40$ are absent; this is due the non-existence of the solution of eq.(16) at such aspect ratios. One can thus conjecture that at temperature $167 \mathrm{~K}$, crystal nuclei of NAT have highly elongated (needle-like, prolate) shape with the aspect ratio greater than 40 .

The thermodynamics and kinetics of crystal nucleation uniquely constrain the size and shape of the critical nucleus with a unique, "native" aspect ratio $\kappa_{*}$. Due to the uncertainties concerning the crystal nucleus morphology, this native aspect ratio $\kappa_{*}$ is not known. Hence, it is impossible to accurately determine the value of $\sigma_{\lambda}^{s v}$. For all temperatures and solution compositions, as $\kappa$ increases by $5000 \%$ (from 1 to 50 ), $\sigma_{\lambda}^{s v}$ slowly increases by less than about $5 \%$ compared to its minimum value at $\kappa=1$. Most estimates suggest $\sigma_{\lambda}^{s v}$ to be a weakly increasing function of $T$. Note that extreme caution should be taken in interpreting these estimates because the experiments on the freezing of large and small droplets were not performed under identical thermodynamic conditions (in violation of the fundamental requirement of eq.(16)). Besides, the scatter of the original experimental rates 
$J_{\text {Large }}^{\text {exp }}$ and $J_{\text {small }}^{\text {exp }}$ would result in large error bars in estimates of $\sigma_{\lambda}^{s v}$ and cause, for example, the non-monotonic dependence of $\sigma^{l s}$ on $T$ in Fig.2b.

One can expect that, more accurate information about the native morphology of crystal nuclei and more self-consistent experimental data sets on $J_{\text {Large }}^{\text {exp }}$ and $J_{\text {small }}^{\text {exp }}$ will eventually allow one to obtain more accurate estimates of $\sigma_{\lambda}^{s v}$ and find its $T$-dependence.

\section{Concluding Remarks}

Using the formalism of classical thermodynamics in the framework of the classical nucleation theory, we have, with as few assumptions as possible, derived an expression for the reverisble work $W_{*}$ of formation of a binary crystal nucleus in a liquid binary solution of non-stoichiometric composition (incongruent crystallization). In applications to the crystallization of aqueous nitric acid droplets, the new expression more adequately takes account of the effects of nitric acid vapors compared to the conventional, widely used expression of MacKenzie et al. ${ }^{26}$

Via numerical calculations, we have compared the predictions of the MKLV and modified expressions for the average liquid-solid interfacial tension $\sigma^{\text {ls }}$ of NAD crystals by applying them to the analysis of existing experimental data on the incongruent crystallization of aqueous nitric acid droplets of composition relevant to PSCs.

It has been shown that the MKLV-expression-based predictions for $\sigma^{\text {ls }}$ are higher by about $5 \%$ compared to the predictions obtained by using the modified expression. Note that $5 \%$ difference in the interfacial tension $\sigma^{l s}$ is quite significant. Indeed, let us consider the volume-based crystal nucleation in liquids. In CNT, the rate of nucleation $J$ is proportional to the exponential of $W_{*}$, that is, $J \sim \exp -W_{*} / k_{B} T$; in turn, $W_{*}$ is proportional to the third power of the surface tension $\sigma^{l s}$, i.e., $W_{*} \sim\left(\sigma^{l s}\right)^{3}$, see eqs.(7) and (8). In order to have a more or less significant nucleation rate (i.e., $J>1 \mathrm{~cm}^{-3} \mathrm{~s}^{-1}$, typical values for $W_{*}$ are usually of the order of $30-50$ thermal units $k_{B} T$ (see, 
e.g., refs.23,24). Thus, one can easily show, that the difference of $5 \%$ in the surface tension $\sigma^{l s}$ would lead to the difference of two orders of magnitude in theoretical predictions for $J$. If for example, atmospheric models predicted the crystal nucleation rate $J$ to be about $10^{5} \mathrm{~cm}^{-3} \mathrm{~s}^{-1}$ based on the MKLV expression for $W_{*}$, then they would now predict $J$ to be about $10^{7} \mathrm{~cm}^{-3} \mathrm{~s}^{-1}$ based on our modified expression for $W_{*}$.

These differences also transpire in the predictions of both expressions for the solid-vapor interfacial tension $\sigma^{\text {sv }}$ of NAD crystal nuclei which can be evaluated from experimental data on crystal nucleation rates $J^{\exp }$ in aqueous nitric acid droplets. To extract $\sigma^{\text {sv }}$ from data on $J^{\text {exp }}$, it is necessary to measure the latter both in small droplets (of radii $R \lesssim 5 \mu \mathrm{m}$ ), wherein the surface-stimulated mode of crystal nucleation is dominant, and in large ones (of radii $R \gtrsim 20 \mu \mathrm{m}$ ), wherein the surface-stimulated mode of crystal nucleation is negligible (compared to the volume-based one); except for the linear size, both small and large droplets must be under identical thermodynamic conditions. The crucial idea of the method is that, even in the surface-stimulated mode, when a crystal nucleus forms with one of its facets constituting a part of the droplet surface, it initially emerges (as a sub-critical cluster) homogeneously in the sub-surface layer, not "pseudo-heterogeneously" at the surface.

Applying that method for determining $\sigma^{\text {sv }}$ to existing ${ }^{16,25}$ experimental data, our expression for $W_{*}$ provides an estimate for $\sigma^{\text {sv }}$ of NAD to be in the range from about $92 \mathrm{dyn} / \mathrm{cm}$ to about 101 dyn/cm, while the MKLV expression predicts it to be in the range from about $95 \mathrm{dyn} / \mathrm{cm}$ to about $105 \mathrm{dyn} / \mathrm{cm}$. Both expressions for $W_{*}$ become identical for the case of congruent crystallization; this was also demonstrated by applying our method for determining $\sigma^{\text {sv }}$ to the NAT crystal nucleation in aqueous nitric acid droplets of stoichiometric composition. We obtained physically sound and well-behaved estimates of the solid-vapor interfacial tension of NAT crystals, from about $95 \mathrm{dyn} / \mathrm{cm}$ to about 110 in the temperature range from $163.5 \mathrm{~K}$ to about $167 \mathrm{~K}$. 


\section{References}

${ }^{1}$ S. Solomon, Stratospheric ozone depletion: A review of concepts and history. Rev. Geophys. 37, 275-316 (1999).

${ }^{2}$ D. Lowe and R. MacKenzie, Review of Polar Stratospheric Cloud Microphysics and Chemistry. J. Atmos. Solar-Terrest Phys. 70, 13-40 (2008).

${ }^{3}$ S. Solomon, The mystery of the Antarctic Ozone "Hole". Rev. Geophys. 26, 131-148 (1988).

${ }^{4}$ M. Völmer, Kinetik der Phasenbildung (Teodor Steinkopff, Dresden und Leipzig, 1939).

${ }^{5}$ D. Turnbull and J.C. Fisher, Rate of Nucleation in Condensed Systems. J. Chem. Phys. 17, 71-73 (1949).

${ }^{6}$ H. R. Pruppacher and J. D. Klett., Microphysics of clouds and precipitation. (Kluwer Academic Publishers, Norwell, 1997).

${ }^{7}$ A. Tabazadeh, Y. S. Djikaev, P. Hamill, and H. Reiss, Laboratory evidence for surface nucleation of solid polar stratospheric cloud particles. J. Phys. Chem. A 106, 10238-10246 (2002).

${ }^{8}$ A. Tabazadeh, Y. S. Djikaev, and H. Reiss, Surface crystallization of supercooled water in clouds. Proc. Natl. Acad. Sci. USA 99, 15873 (2002).

${ }^{9}$ Y. S. Djikaev, A. Tabazadeh, P. Hamill, and H. Reiss, Thermodynamic conditions for the surfacestimulated crystallization of atmospheric droplets. J.Phys.Chem. A 106, 10247 (2002).

${ }^{10}$ Y. S. Djikaev, A. Tabazadeh, and H. Reiss, Thermodynamics of crystal nucleation in multicomponent droplets: Adsorption, dissociation, and surface-stimulated nucleation. J.Chem.Phys. 118, 6572-6581 (2003). 
${ }^{11}$ R. Defay, I. Prigogine, A. Bellemans, and D. H. Everett, Surface Tension and Adsorption (John Wiley, New York, 1966).

${ }^{12}$ B. Mutaftschiev, and J. Zell, Interfacial energy and cadmium growth in molten baths. Surf. Sci. 12, 317 (1968).

${ }^{13}$ G. Grange, R. Landers, and B. Mutaftshiev, Contact angle and surface morphology of KCl crystalmelt interface studied by bubble-method Surf. Sci. 54, 445-462 (1976).

${ }^{14}$ D. Chatain and P. Wynblatt, in Dynamics of Crystal Surfaces and Interfaces, Ed. P.M.Duxbury and T.J.Pence, 53-58 (Springer, NY, 2002).

${ }^{15}$ M. Elbaum, S. G. Lipson, and J. G. Dash, Optical study of surface melting on ice. J. Cryst. Growth 129, 491-505 (1993).

${ }^{16}$ N. H. Fletcher, The physics of rainclouds. (University Press, Cambridge, 1962).

${ }^{17}$ E. Ruckenstein and G. Berim, Kinetic theory of nucleation. (New York, 2016).

${ }^{18}$ Y. S. Djikaev, Effect of the Surface-Stimulated Mode on the Kinetics of Homogeneous Crystal Nucleation in Droplets. J. Phys. Chem. A 112, 6592-6600 (2008).

${ }^{19}$ T. Kuhn, M. E. Earle, A. F. Khalizov, and J. J. Sloan, Size dependence of volume and surface nucleation rates for homogeneous freezing of supercooled water droplets. Atmos. Chem. Phys. 11, 2853-2861 (2011).

${ }^{20}$ O. Stetzer, O. Möhler, R. Wagner, S. Benz, H. Saathoff, H. Bunz, and O. Indris, Homogeneous nucleation rates of nitric acid dihydrate (NAD) at simulated stratospheric conditions - Part I: Experimental results. Atmos. Chem. Phys. 6, 3023-3033 (2006). 
${ }^{21}$ R .S. Disselkamp, S. E. Anthony, A. J. Prenni, T. B. Onasch, and M. A. Tolbert, Crystallization kinetics of nitric acid dihydrate aerosols, J. Phys. Chem. 100, 9127-9137 (1996).

${ }^{22}$ A. R. MacKenzie, M. Kulmala, A. Laaksonen, T. Vesala, On the theories of type 1 polar stratospheric cloud formation. J. Geophys. Res. 100, 11275-11288 (1995).

${ }^{23}$ A. J. Prenni, T. B. Onasch, R. T. Tisdale, R. L. Siefert, and M. A. Tolbert, Composition-dependent freezing nucleation rates for HNO3/H20 aerosols resembling gravity-perturbed stratospheric particles. J. Geophys. Res. 103, 28439-28450 (1998).

${ }^{24}$ R. T. Tisdale, A. M. Middlebrook, A. J. Prenni, and M. A. Tolbert, Crystallization kinetics of HNO3/H20 films representative of polar stratospheric clouds. J. Phys. Chem. A 101, 21122119 (1997).

${ }^{25}$ D. Salcedo, L. T. Molina, and M. J. Molina, Homogeneous freezing of concentrated aqueous nitric acid solutions at polar stratospheric temperatures. J. Phys. Chem. A 105, 1433-1439 (2001).

${ }^{26}$ A. R. MacKenzie, M. Kulmala, A. Laaksonen, and T. Vesala, Correction to "On the theories of type 1 polar stratospheric cloud formation" by A.R.Mackenzie, et al. 102, 19729-19730 (1997).

${ }^{27}$ A. K. Bertram and J. J. Sloan, The nucleation rate constants and freezing mechanism of nitric acid trihydrate aerosol under stratosphefic conditions, J. Geophys. Res. 103, 13,261-13,265 (1998).

${ }^{28}$ A. Bogdan, M. J. Molina, H. Tenhu, E. Mayer, T. Loerting, Formation of mixed-phase particles during the freezing of polar stratospheric ice clouds. Nature Chemistry 2 (3), 197-201 (2010).

${ }^{29}$ A. Bogdan and M. J. Molina, Why Does Large Relative Humidity with Respect to Ice Persist in Cirrus Ice Clouds? J. Phys. Chem. A 113, 14123-14130. 
${ }^{30}$ D. J. Lee, M. M. Telo da Gama, and K. E. Gubbins, A micrsoscopic theory for spherical interfaces: Liquid drops in the canonical ensemble. J. Chem. Phys. 85, 490-499 (1986).

${ }^{31}$ Y. Djikaev and E. Ruckenstein, A kinetic model for the premelting of a crystalline structure, Physica A 387, 134-144 (2008).

${ }^{32}$ X. Wei, P. B. Miranda, and Y. R. Shen, Surface vibrational spectroscopic study of surface melting of ice. Phys. Rev. Lett. 86, 1554-1557 (2001).

${ }^{33}$ X. Wei and Y. R. Shen, Vibrational spectroscopy of ice interfaces. Applied Phys. B 74, 617-620 (2002).

(34)S. Toxvaerd, N. Larsen, and J. C. Dyre, Simulations of crystallization in supercooled nanodroplets in the presence of a strong exothermic solute: The mystery of the Antarctic ozone "hole". J. Phys. Chem. C 115, 12808-12814 (2011).

${ }^{35}$ B. Luo, K. S. Carslaw, T. Peter, and S. L. Clegg, Vapour pressures of H2SO4/HNO3/HCl/HBr/H2O solutions to low stratospheric temperatures. Geophys. Res. Lett. 22, 247-250 (1995).

${ }^{36}$ E. Martin, C. George, and P. Mirabel, Densities and Surface Tensions of H2SO4/HNO3/H20 solutions. Geophys. Res. Lett. 27, 197-200 (2000).

${ }^{37}$ P. J. Wooldridge, R. Zhang, and M. J. Molina, Phase equilibria of H2SO4, HNO3, and HCl hydrates and the composition of polar stratospheric clouds. Geophys. Res. Lett. 100, 1389-1396 (1995).

${ }^{38}$ V. Granzhan and S. Laktionova, The densities, viscosities, and surface tensions of aqueous nitric acid solutions. Russian J. Phys. Chem. 49, 1448 (1975).

${ }^{39}$ R. Wagner, O. Möhler, H. Saathoff, O. Stetzer, and U. Schurath, Infrared Spectrum of Nitric Acid Dihydrate: Influence of Particle Shape. J. Phys. Chem. A 109, 2572-2581 (2005). 
${ }^{40}$ H. Grothe, H. Tizek, D. Waller, and D. J. Stokes, The crystallization kinetics and morphology of nitric acid trihydrate. Phys. Chem. Chem. Phys. 8, 2232-2239 (2006). 


\section{Captions}

to Figures 1 to 5 of the manuscript "Free EnERGy of FORMATion of A CRYSTAL NuCleus in INCONGRUENT SOLIDIFICATION: IMPLICATION FOR MODELING THE CRYSTALLIZATION OF AQUEOUs Nitric ACID DROplets in POlar Stratospheric Clouds" by Yuri S. Djikaev and Eli Ruckenstein.

Figure 1. Typical dependence of the RHS of eq.(14) on the variable $u$.

Figure 2. The $T$-dependence of the effecitve liquid-solid interfacial tension $\sigma_{\lambda}^{l s}$ of NAD crystal nuclei obtained by using the parameterized dependence ${ }^{25}$ of $J_{\text {Large }}^{\text {exp }}$ on $T$ and wt $\% \mathrm{HNO}_{3}$ for the nonstoichiometric mole fractions of $\mathrm{HNO}_{3}$ in large aqueous NA droplets: a) $\chi=0.20$; b) $\chi=0.30$. The solid and dashed curves correspond to $\sigma_{\mathrm{DR}}^{l s}$ (given by eq.(9)) and $\sigma_{\mathrm{MKLV}}^{l s}$ (given by eq.(10)), respectively.

Figure 3. The $\chi$-dependence of the effecitve liquid-solid interfacial tension $\sigma_{\lambda}^{l s}$ of NAD crystal nuclei obtained by using the parameterized dependence ${ }^{25}$ of $J_{\text {Large }}^{\text {exp }}$ on $T$ and wt $\% \mathrm{HNO}_{3}$, for large aqueous NA droplets of non-stoichiometric composition at a) $T=190 \mathrm{~K}$ and b) $T=197 \mathrm{~K}$. The solid and dashed curves correspond to $\sigma_{\mathrm{DR}}^{l s}$ (given by eq.(9)) and $\sigma_{\mathrm{MKLV}}^{l s}$ (given by eq.(10)), respectively.

Figure 4. The $\kappa$-dependence of the solid-vapor interfacial tension $\sigma_{\lambda}^{s v}(\mathrm{dyn} / \mathrm{cm})$ of facet $\lambda$ of NAD crystal nuclei due to the experimental data from ref.25 (large aqueous NA droplets) and ref.20 (small aqueous NA droplets). The results in Fig.4a were obtained by using eqs.(9) and (17), whereas those in Fig.4b were obtained by using eqs.(10) and (17). Circles represent experiments on droplets of mean diameter $225 \mathrm{~nm}$ and NA mole fraction 0.27 at temperature $195.8 \mathrm{~K}$; squares are for droplets of mean diameter $255 \mathrm{~nm}$ and NA mole fraction 0.27 at temperature $192.2 \mathrm{~K}$; diamonds are for droplets of mean diameter $255 \mathrm{~nm}$ and NA mole fraction 0.28 at temperature $192.1 \mathrm{~K}$. 
Figure 5. The $\kappa$-dependence of the solid-vapor interfacial tension $\sigma_{\lambda}^{s v}(\mathrm{dyn} / \mathrm{cm})$ of facet $\lambda$ of NAT crystal nuclei due to the experimental data of ref.25 (large aqueous NA droplets of mean radius $25 \mu \mathrm{m}$ and stoichiometric NAT compositions, 54 wt\% $\mathrm{HNO}_{3}$ ) and ref.27 (small aqueous NA droplets of mean radius $0.38 \mu \mathrm{m}$ and NAT composition of $53.7 \mathrm{wt} \% \mathrm{HNO}_{3}$ ). Circles correspond to the experiments ${ }^{27}$ at temperature $163.5 \mathrm{~K}$, the squares at $165.5 \mathrm{~K}$, and diamonds at $167 \mathrm{~K}$. 


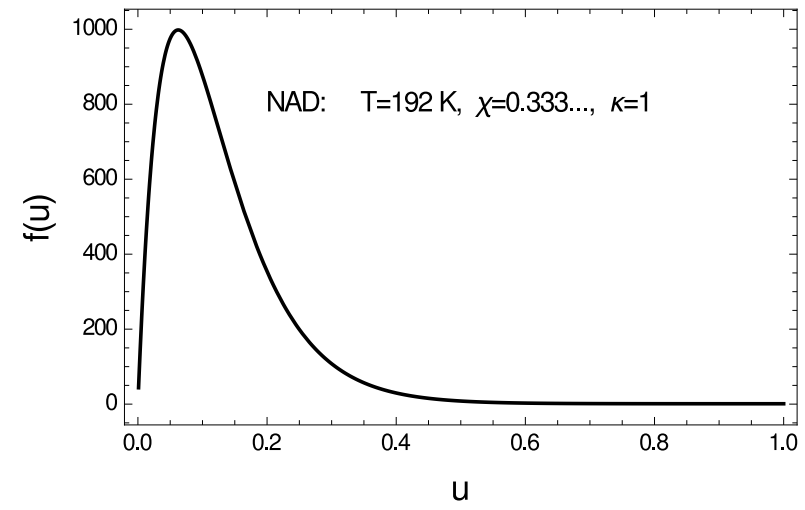

Figure 1: 
a)
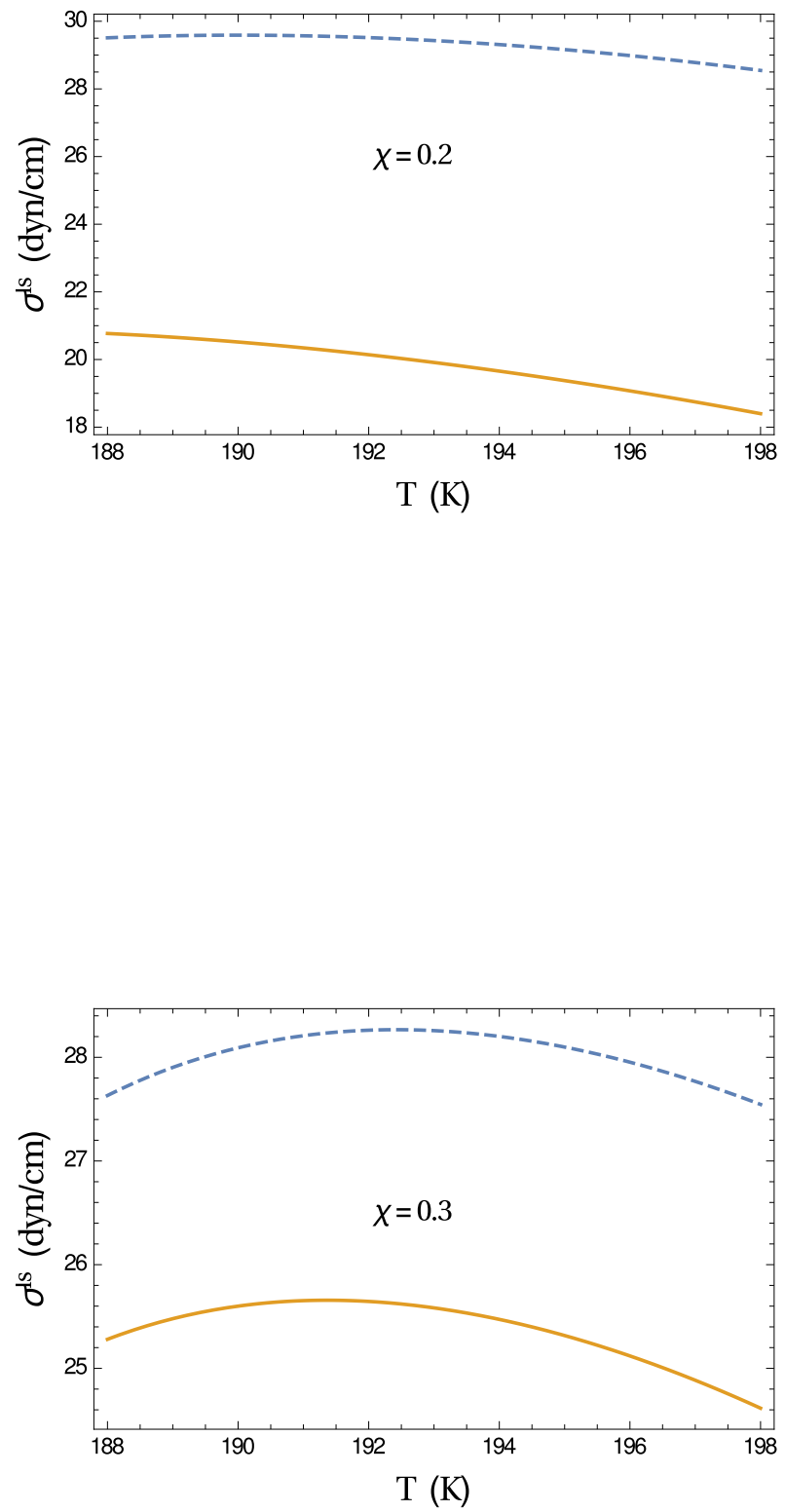

Figure 2: 


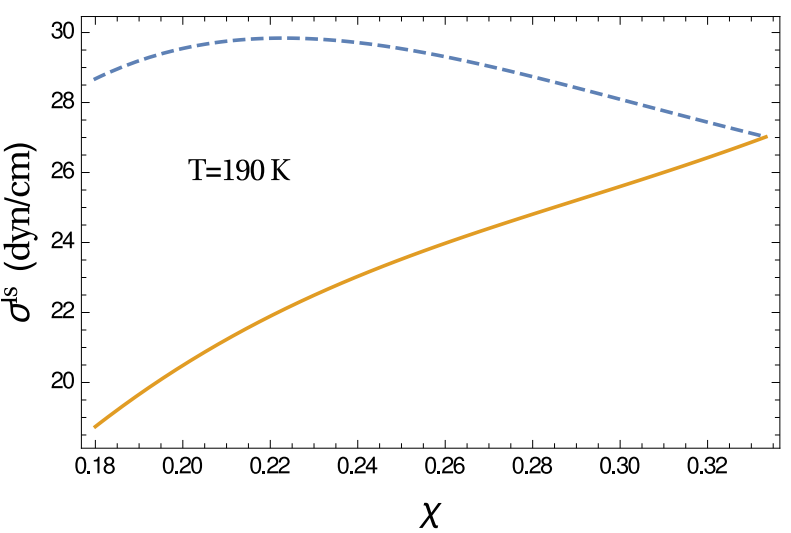

a)

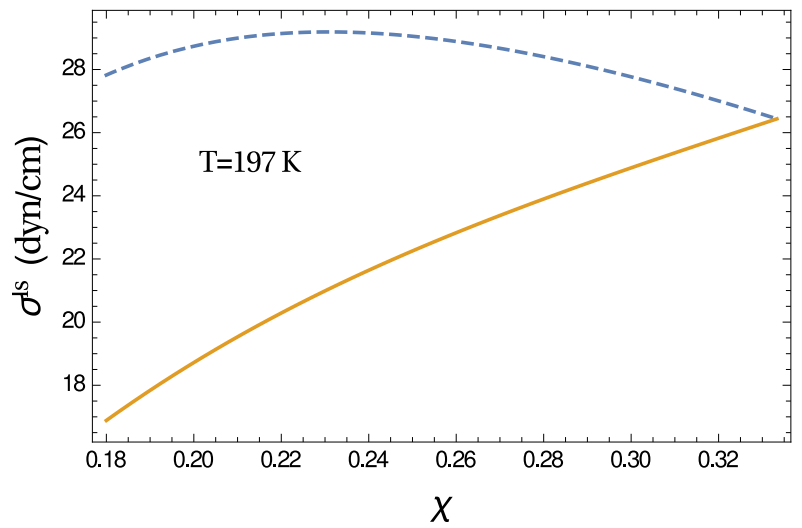

Figure 3: 


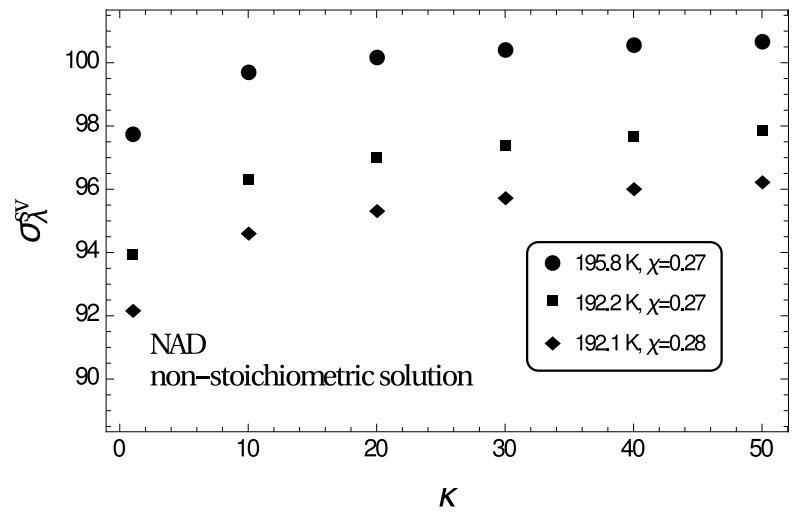

a)

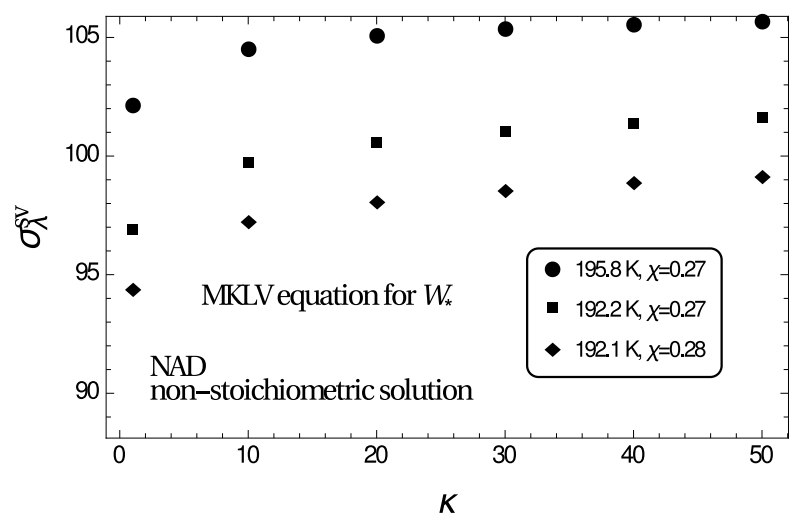

b)

Figure 4: 


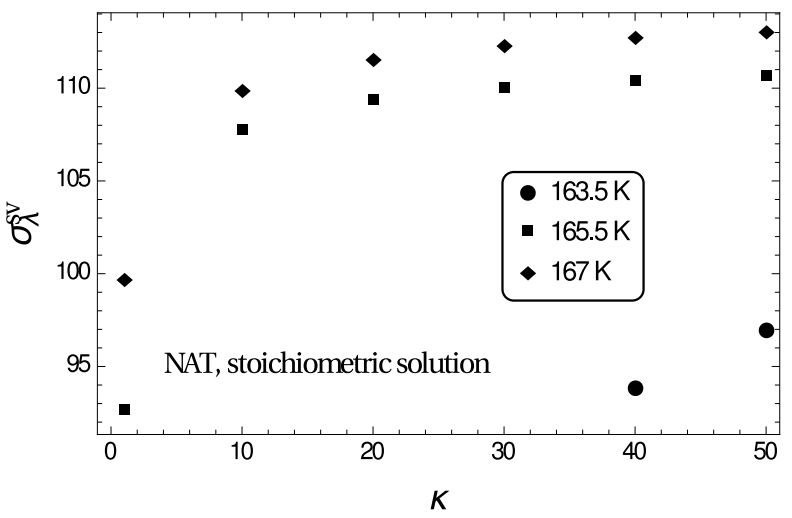

Figure 5: 